\title{
Relationship Between Duration of Type 2 Diabetes and Effectiveness of DPP-4 Inhibitor Versus Sulfonylurea as Add-on Therapy: A Post Hoc Analysis
}

\author{
Helmut Brath · Päivi M. Paldánius · Giovanni Bader · Chantal Mathieu
}

Received: March 31, 2017 / Published online: June 19, 2017

(C) The Author(s) 2017. This article is an open access publication

\begin{abstract}
Introduction: To assess the impact of duration of type 2 diabetes on glucose-lowering effectiveness of the dipeptidyl peptidase-4 (DPP-4) inhibitor vildagliptin versus sulfonylureas (SUs) in a real-life setting.

Methods: Data were extracted from the large 1-year, observational EDGE study $(N=45,868)$. Patients receiving either DPP-4 inhibitor or any SU as add-on to monotherapy were selected $(N=36,164)$. Impact of the disease duration on change in glycated hemoglobin (HbA1c) levels was evaluated by using a linear multiple regression model. Descriptive statistics assessed the proportion of patients achieving the composite endpoint (HbA1c $<7.0 \% ; 53.0 \mathrm{mmol} / \mathrm{mol}$ without hypoglycemia or weight gain), stratified by diabetes duration.
\end{abstract}

Results: At baseline, the overall mean $( \pm \mathrm{SD})$ type 2 diabetes duration was $5.4 \pm 5.24$ years, and

Enhanced content To view enhanced content for this article go to http://www.medengine.com/Redeem/ 1898F0603F3D4859.

H. Brath $(\bowtie)$

Health Center South, Vienna, Austria

e-mail: helmut.brath@gmail.com

P. M. Paldánius · G. Bader

Novartis Pharma AG, Basel, Switzerland

C. Mathieu

Catholic University of Leuven, Leuven, Belgium
HbA1c was $8.2 \pm 1.33 \%(66.0 \pm 14.5 \mathrm{mmol} / \mathrm{mol})$. HbA1c lowering was directly proportional to the baseline HbA1c ( -0.69 per unit; 95\% CI -0.696 , $-0.681 ; p<0.0001)$ and inversely proportional to the disease duration ( 0.01 per year; $95 \%$ CI 0.01 , $0.013)$. There was an increased loss of $\beta$-cell function (less pronounced HbA1c drop with increasing disease duration) in patients treated with SU-based regimens $(0.025$; 95\% CI 0.022, 0.027) compared with vildagliptin-based regimens $(0.005 ; 95 \%$ CI $0.003,0.007)$, with the mean adjusted difference being 0.10 (95\% CI -0.122 , $-0.092 ; p<0.0001)$. Consistently, a higher proportion of patients achieved the composite endpoint with vildagliptin over the diabetes duration (less than 2 to more than 10 years).

Conclusion: Vildagliptin demonstrated less dependency on the duration of type 2 diabetes, whereas the effectiveness of SUs diminished faster with increasing duration of the disease in a real-life setting.

Funding: Novartis Pharma AG.

Keywords: Diabetes duration; Glycemic; HbA1c; Real-life; Sulfonylurea; Type 2 diabetes; Vildagliptin

\section{INTRODUCTION}

The progressive decline in $\beta$-cell function in type 2 diabetes entails the use of combination therapy with different glucose-lowering agents 
on top of lifestyle interventions to maintain glycemic control as duration of disease increases [1]. According to the American Diabetes Association/European Association for the Study of Diabetes position statement, treatment choices should be individualized on the basis of patients' needs as well as effectiveness, tolerability, and long-term safety of antihyperglycemic agents [1]. When first-line monotherapy (mostly metformin) is insufficient to maintain glycemic control, initiation of second-line treatment is needed, with commonly chosen alternatives being dipeptidyl peptidase-4 (DPP-4) inhibitors and sulfonylureas (SUs), based on their $\beta$-cell-targeting properties $[1,2]$. SUs exhibit effective glucose-lowering capability, albeit at the cost of an increased risk of hypoglycemia, caused by its glucose-independent mechanism of action, along with weight gain $[1,2]$. DPP-4 inhibitors ameliorate glucose homeostasis by increasing both the $\alpha$ and $\beta$-cell responsiveness to glucose, leading to improvements in $\alpha$ - and $\beta$-cell functions in patients with type 2 diabetes [3]. Randomized clinical trials (RCTs) have demonstrated the translation of these effects into good efficacy and safety profile of the DPP-4 inhibitors, with low risk of hypoglycemia and weight gain [4-7].

Data obtained from RCTs are considered the gold standard; however, it requires supplementation with data from real-life studies to comprehend the real potential of the individual glucose-lowering drugs $[8,9]$. Despite meeting the regulatory and scientific standards, selective population and intensive clinical supervision sometimes limit RCTs from being designed to contemplate situations in routine clinical practice [8-10]. Real-life outcome studies play a pivotal role in evaluating treatment effectiveness and aftermaths, especially in large heterogeneous patient populations, without stringent inclusion and exclusion criteria or even other aspects that limit generalizability of results from RCTs $[8,9]$. A recent analysis reported differences in glycated hemoglobin (HbA1c)-lowering effectiveness with SU treatment in real-life versus RCTs [8]. Data from A Diabetes Outcome Progression Trial (ADOPT) and the United Kingdom Prospective Diabetes Study (UKPDS) are indicative of uncertainty with SU treatment in maintaining good glycemic control over a long period of time $[11,12]$. DPP-4 inhibitors, on the contrary, retain treatment effectiveness by demonstrating comparable $\mathrm{HbA1c}$ reductions in both real-life setting and RCTs [4-8], thereby emphasizing an increasing need for pragmatic trials [10].

Accordingly, the present post hoc analysis from the Effectiveness of Diabetes control with vildaGliptin/mEtformin (EDGE) study [13] aimed to evaluate the impact of the duration of type 2 diabetes on the glucose-lowering effectiveness of DPP-4 inhibitor- and SU-based regimens, in a real-life setting.

\section{METHODS}

\section{Patient Population}

EDGE was a 1-year, prospective, real-life, observational study that reported the effects of second-line oral antidiabetes drugs (OADs) on 45,868 patients with type 2 diabetes from 2957 centers across 27 countries worldwide. The study enrolled men and women aged greater than 18 years with type 2 diabetes, having inadequate glycemic control with monotherapy and were prescribed a new add-on OAD (either vildagliptin or vildagliptin/metformin, or drugs including sulfonylureas, metformin, thiazolidinediones, glinides, or $\alpha$-glucosidase inhibitors, or an OAD fixed-dose combination) as per clinical judgment by the investigators. Patients provided necessary verbal or written informed consent, as defined by the national regulations. Patients meeting required inclusion criteria became eligible only after the treatment decision was finalized by the physician. Patients were then assigned to either the DPP-4 inhibitor (dose as per label) or comparator OADs. A drug-naïve patient or any individual using insulin, incretin mimetics, or any investigational drug at the time of enrollment, and those with a history of hypersensitivity to any of the study drugs, was excluded from the study. Additional details on the selected patient population and inclusion/exclusion criteria are available in the primary published manuscript [13]. Sodium glucose co-transporter-2 (SGLT-2) 
inhibitors were not included in the EDGE study as part of comparator OADs since they were not available on the market. Moreover, when EDGE study enrolled patients, the use and accessibility of comparator OADs varied greatly across 27 countries worldwide. Further details of the study design are reported in the primary publication [13]. In this post hoc analysis, evaluable patients (with available HbA1c values) who were assigned to either of the two most frequently chosen regimens, vildagliptin- or SU-based, were analyzed. We have adjusted the analysis on the basis of prior presumed factors which might influence response to any OADs in patients with shorter or longer duration of diabetes.

\section{Study Assessments}

Impact of the duration of type 2 diabetes on change $(\Delta)$ in HbA1c from baseline to the end of the treatment (12 months) was the primary assessment. The proportion of patients achieving the composite endpoint (HbA1c $<7.0 \%$; $53.0 \mathrm{mmol} / \mathrm{mol}$ ) without hypoglycemia and weight gain $(\geq 3 \%)$, stratified by the disease duration, was evaluated as a secondary endpoint. These primary and secondary assessment parameters were arbitrarily chosen among potential confounders of the association between HbA1c drop and duration of the treatment.

\section{Statistical Analysis}

To explore the role of duration of diabetes on the change in HbA1c at 12 months for this post hoc analysis, a few more potential predictors were selected a priori on the basis of availability and known effect on HbA1c change. The complex interplay between the $\Delta$ in HbA1c at 12 months and baseline HbA1c, duration of type 2 diabetes, and body mass index (BMI) was derived by means of a linear multiple regression model. A second model with an interaction term was built specifically to assess the relationship between the duration of type 2 diabetes and treatment regimen (DPP-4 inhibitor and $\mathrm{SU})$. For further understanding of the clinical impact of the treatment on the interplay between duration of diabetes and change in $\mathrm{HbA} 1 \mathrm{c}$, a combined endpoint (HbA1c $<7.0 \%$; $53.0 \mathrm{mmol} / \mathrm{mol}$, no hypoglycemia or no weight gain) was also evaluated. The $\Delta$ in HbA1c and body weight were analyzed by using descriptive statistics.

\section{Compliance with Ethical Guidelines}

This study is a subanalysis of primary EDGE publication. The study protocol for EDGE was reviewed and approved by all local independent ethics committees or institutional review boards (IRBs). All procedures followed were in accordance with the ethical standards of the committee on human experimentation and with the Declaration of Helsinki of 1964, as revised in 2013, and Good Clinical Practice Guidelines. Written informed consent was obtained from all patients prior to inclusion in the study.

\section{RESULTS}

The study comprised 36,164 patients receiving dual therapy: 24,721 received vildagliptin-based and 11,443 received SU-based regimen. Patient demographics and baseline characteristics are summarized in Table 1. At baseline, the overall mean $( \pm \mathrm{SD}) \quad \mathrm{HbA} 1 \mathrm{c}$ was $8.2 \pm 1.33 \%$ $(66.0 \pm 14.5 \mathrm{mmol} / \mathrm{mol})$, mean duration of type 2 diabetes was $5.4 \pm 5.24$ years, and BMI was $29.1 \pm 5.20 \mathrm{~kg} / \mathrm{m}^{2}$. The mean baseline weight was numerically higher in patients treated with vildagliptin $(82.1 \mathrm{~kg})$ versus $S U(77.2 \mathrm{~kg})$, and of those patients receiving dual therapy in general, more men received SU (54.3\%) or vildagliptin $(55.0 \%)$.

The $\Delta$ in HbA1c from baseline was directly proportional to the baseline HbA1c $(-0.69$ per unit; 95\% CI $-0.696,-0.681 ; p<0.0001)$ and inversely proportional to the disease duration (0.01 per year; 95\% CI 0.01, 0.013; $p<0.0001$ ) as well as BMI (0.008 per unit; 95\% CI 0.006, $0.0102 ; p<0.0001)$. The mean reduction in HbA1c levels from baseline was consistently higher in vildagliptin-treated than SU-treated patients. The $\Delta$ in HbA1c between vildagliptin 
Table 1 Patient demographics and baseline characteristics

\begin{tabular}{llll}
\hline Characteristics & $\begin{array}{l}\text { Vildagliptin-based }^{\mathbf{a}} \\
(\boldsymbol{n}=\mathbf{2 4 , 7 2 1})\end{array}$ & $\begin{array}{l}\text { SU-based }^{\mathbf{a}} \\
(\boldsymbol{n}=\mathbf{1 1 , 4 4 3})\end{array}$ & $\begin{array}{l}\text { Total } \\
(\boldsymbol{N}=\mathbf{3 6 , 1 6 4 )}\end{array}$ \\
\hline $\mathrm{HbAlc,} \mathrm{\%} \mathrm{(mmol/mol)}$ & $8.1 \pm 1.4(65.0 \pm 15.3)$ & $8.2 \pm 1.3(66.0 \pm 14.5)$ & $8.2 \pm 1.3(66.0 \pm 14.5)$ \\
$\mathrm{BMI}, \mathrm{kg} / \mathrm{m}^{2}$ & $29.5 \pm 5.3$ & $28.2 \pm 4.8$ & $29.1 \pm 5.2$ \\
Body weight, kg & $82.1 \pm 17.5$ & $77.2 \pm 15.0$ & $80.6 \pm 16.9$ \\
Diabetes duration, years & $5.5 \pm 5.4$ & $5.4 \pm 5.0$ & $5.4 \pm 5.2$ \\
Median, range (min-max) & $4.0(0.0-56.3)$ & $4.0(0.0-74.7)$ & $4.0(0.0-74.7)$ \\
eGFR, mL/min/1.73 m ${ }^{2}$ & $90.6 \pm 49.8$ & $90.1 \pm 51.8$ & $90.4 \pm 50.5$ \\
Men, \% & 55.0 & 54.3 & 55.0 \\
Complications, \% & & & 7.7 \\
Microvascular & 7.6 & 7.8 & 10.9 \\
Macrovascular & 11.2 & 10.2 & \\
Background medications, \% & & & 100.0 \\
Antidiabetic & 100.0 & 100.0 & 4.8 \\
Antihypertensives & 5.5 & 3.2 & 15.3 \\
Diuretics & 16.1 & 13.4 & \\
\hline
\end{tabular}

Data represented as mean \pm SD or $n(\%)$

$B M I$ body mass index, $C V$ cardiovascular, $e G F R$ estimated glomerular filtration rate, $H b A 1 c$ glycated hemoglobin, $S D$ standard deviation, $S U$ sulfonylurea

a Dual regimens with a component of vildagliptin or SU. Total sample size reflects the total number of evaluable patients with an available $\mathrm{HbAlc}$ value

and SU-based regimens was $-0.21 \%(95 \% \mathrm{CI}$ $-0.232 \%,-0.191 \% ; p<0.0001)$.

The adjusted mean $\Delta$ in HbA1c from baseline against diabetes duration is depicted in Fig. 1. A positive correlation was observed between the $\Delta$ in HbA1c from baseline and duration of the disease (adjusted $r^{2}=0.53$, $p<0.0001$ ). The rate of loss of function (less pronounced HbA1c drop with increasing disease duration) for every year of the disease duration was higher ( $\sim 5$ times) with SU-based regimen (0.025; 95\% CI $0.022, \quad 0.027$; $p<0.0001)$ than for vildagliptin-based regimen (0.005; 95\% CI 0.0034, 0.0073; $p<0.0001)$, the adjusted marginal difference being 0.10 (95\% CI $-0.122,-0.0918 ; p<0.0001)$. The proportion of patients achieving HbA1c $<7.0 \%$ $(53.0 \mathrm{mmol} / \mathrm{mol})$ without hypoglycemia or weight gain over a disease duration ranging

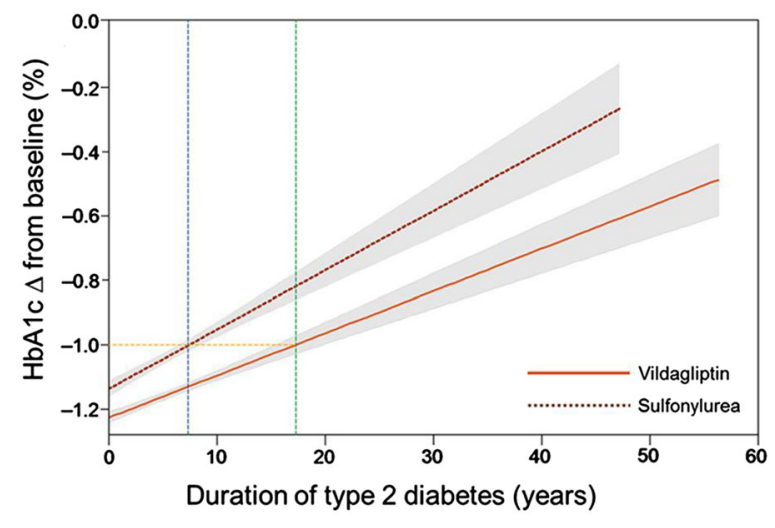

HbAlc, glycated hemoglobin

Fig. 1 Impact of type 2 diabetes duration on mean change $(\Delta)$ in $\mathrm{HbAlc}$ from baseline

from less than 2 to more than 10 years was consistently higher in the vildagliptin versus the SU regimen (Fig. 2). 


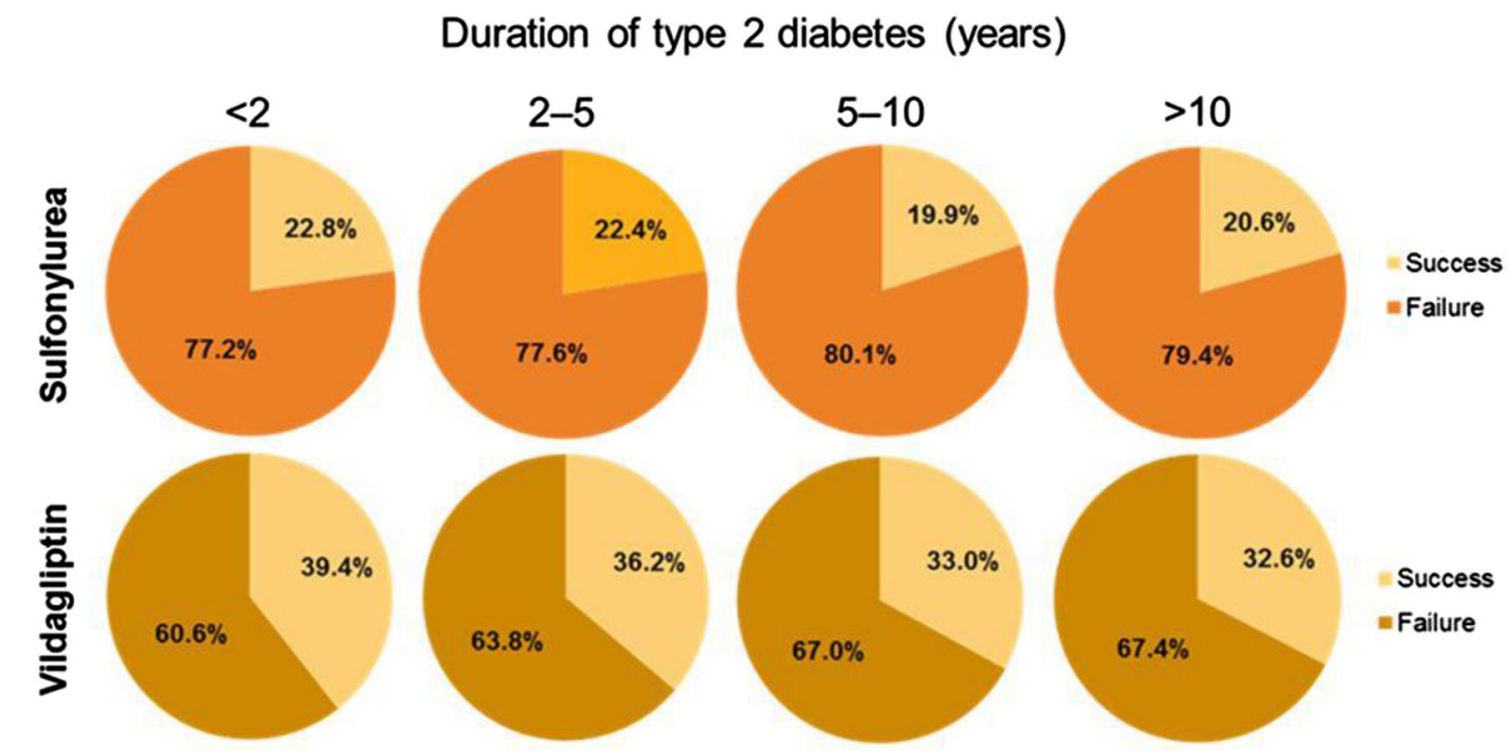

Success: Patients achieving the composite endpoint $[\mathrm{HbAlc}<7.0 \%(53 \mathrm{mmol} / \mathrm{mol})$ without hypoglycemia and weight gain ( $\geq 3 \%$ )]; Failure: patients failing to achieve the composite endpoint.

HbAlc, glycated hemoglobin

Fig. 2 Proportion of patients achieving HbAlc $<7.0 \%(53.0 \mathrm{mmol} / \mathrm{mol})$ without hypoglycemia and weight gain ( $\geq 3 \%)$ stratified by type 2 diabetes duration

\section{DISCUSSION}

This post hoc analysis was conducted to assess the effectiveness of DPP-4 inhibitor vildagliptinversus SU-based regimens in reducing HbA1c levels in relation to the duration of type 2 diabetes. The results showed a more pronounced reduction in $\mathrm{HbA1c}$ with vildagliptin-treated patients compared with SU-treated patients across the duration of the disease in a real-life setting.

Typically individuals develop impaired glucose tolerance as a result of increased insulin resistance, which in turn increases the stress on $\beta$-cells to secrete more insulin to combat the increased peripheral insulin resistance. As the disease progresses, multiple pathophysiological defects such as increased hepatic glucose production, increased insulin resistance, and decrease in $\beta$-cell function due to various putative biological processes including endoplasmic reticulum (ER) stress result in increasing hyperglycemia and HbA1c. Durability of response to an agent will depend on its ability to target the multiple pathophysiological defects. One explanation for durable efficacy of a DPP-4 inhibitor versus SUs may be the progressive $\beta$-cell decline [14] throughout the course of diabetes duration. SUs target deficient insulin secretion by working on $\beta$-cells, whereas glucagon-like peptide-1 (GLP-1)-based medications (DPP-4 inhibitors and GLP-1 receptor agonists) additionally work on the pancreatic $\alpha$-cells, regulating glucagon release, and thereby results in sustained efficacy even at later stages of type 2 diabetes [15]. A study by Kozlovski et al. reported that the response of the $\beta$-cell, but not the HbA1c reduction, with vildagliptin is dependent on the duration of type 2 diabetes. The authors conclude that glycemic durability is maintained with vildagliptin as glucagon suppression may be compensating the reduced $\beta$-cell function [16]. Furthermore, there are reports which suggest that GLP-1 receptor activation improves survival of $\beta$-cells exposed to chemically induced ER stress [17].

In this analysis, we found superiority in efficacy with the DPP-4 inhibitor at all stages of 
the disease duration, after an observational period of 12 months. The reason might be that the treatment effectiveness is retained in reallife with the DPP-4 inhibitor, whereas the same is not observed in SUs, possibly because of the fear of hypoglycemia and thus not always the optimal recommended doses are being used [8]. Our study contributes to the knowledge that the difference in efficacy between SUs and the DPP-4 inhibitor vildagliptin increases over the years of diabetes duration, favoring DPP-4 inhibitors. The choice of an efficient second-line OAD might prevent further intensification to a triple therapy or to a therapy with injectable antidiabetes medication. On the contrary, a retrospective, cohort study reported better durability of glycemic response (reaching HbA1c $<7.0 \%$ ) with SU as add-on therapy in patients failing on metformin monotherapy versus DPP-4 add-on. The disparity in the results can be attributed to differences in baseline HbA1c and duration of diabetes which are strong drivers of the response [18].

Our secondary endpoint, reaching the goal of HbA1c $<7.0 \%(53.0 \mathrm{mmol} / \mathrm{mol})$ without hypoglycemia or weight gain, also demonstrated better efficacy and tolerability with vildagliptin, when compared with SUs, over the course of the disease duration. This might be of particular importance for the elderly population and for patients with renal impairment. Both groups often have prolonged duration of type 2 diabetes in general and are more vulnerable to adverse side effects, especially hypoglycemia [19]. Furthermore, the favorable extrapancreatic effects of DPP-4 inhibitors such as decrease in blood pressure and improvement in lipid profile which positively influences various diabetic complications are also important clinical considerations when choosing an appropriate second-line agent [20-22].

Despite a substantial decline in diabetes-related complications in the past two decades [23], the continued increase in the prevalence of type 2 diabetes is alarming [24]. Furthermore, with the age of disease manifestation being shifted to younger years, we are likely to see more patients with a prolonged duration of diabetes. Hence it is of utmost importance to know about efficacy of different diabetes medications, preferably without tolerability issues.
This study demonstrates that with increased duration of type 2 diabetes, the effectiveness of SUs decreases significantly compared to DPP-4 inhibitor and that a higher percentage of patients on DPP-4 inhibitor are able to reach their glycemic/HbA1c targets without hypoglycemia or weight gain.

The large patient population $(N=36,164)$ and real-life setting are the key strengths of this study. Another strength is the comparison of two clinically, often used second-line therapies, DPP-4 inhibitor and SUs. The current analysis accentuates the fact that the variance in treatment effectiveness between SU and DPP-4 inhibitor in reducing $\mathrm{HbA1c}$ with increasing duration of type 2 diabetes might be pronounced in patients in real-life, adding one more factor in facilitating clinical judgment. This could be of special importance to individuals with a prolonged duration of type 2 diabetes and/or for the elderly population with diabetes.

This paper has certain limitations: We compared only the DPP-4 inhibitor vildagliptin with SUs. Effectiveness of other OADs, especially SGLT-2 inhibitors, over the course of diabetes duration has not been reported as SGLT-2 inhibitors were not on the market during EDGE study initiation. The duration of type 2 diabetes is based on verbally reported values by the patients, and cannot be confirmed; hence, it may not truly reflect the actual diabetes duration. Another limitation of the current study is the fact that baseline BMI affects the change in HbA1c, albeit minimally, and was not taken into account while assessing the impact of diabetes duration on the HbA1c-lowering potential of vildagliptin- and SU-based regimens.

\section{CONCLUSIONS}

This post hoc analysis of a large real-life cohort from the EDGE study demonstrates a distinct reduction of efficacy of oral antidiabetes medication with duration of type 2 diabetes. The $\mathrm{HbA1c}$-lowering potential of SU diminished at a faster rate with increasing duration of type 2 diabetes as a result of progressive deterioration in $\beta$-cell function when compared to the DPP-4 inhibitor vildagliptin. 


\section{ACKNOWLEDGEMENTS}

Sponsorship and article processing charges for this study were funded by Novartis Pharma AG.

All authors had access to the data in this study and thereby take complete responsibility for the data integrity and accuracy of the data analysis. All authors have met the International Committee of Medical Journal Editors (ICMJE) criteria for authorship of this manuscript and have given final approval for the version to be published. The authors would like to thank Rangan Gupta and Amit Kumar Garg of Novartis Healthcare Private Limited, Hyderabad, India, for medical writing and editorial support.

All authors contributed to the conception, design, and interpretation of the analysis. Giovanni Bader contributed towards statistical analyses. Helmut Brath drafted the first draft of the manuscript; Päivi M. Paldánius, Giovanni Bader, and Chantal Mathieu helped with critical review and finalization of the manuscript. All the authors had full access to the data and assume responsibility for the integrity of the data and approved the final manuscript for submission.

Disclosures. Helmut Brath has received honoraria for lectures and advisory board/ speaker's bureau fees from all major diabetes companies, including Novartis. Päivi M. Paldánius is an employee and shareholder of Novartis. Giovanni Bader is an employee and shareholder of Novartis. Chantal Mathieu has received advisory board fees and research support from Novo Nordisk, Sanofi, Merck Sharp \& Dohme, Eli Lilly \& Company, Bristol Myers Squibb, AstraZeneca, Pfizer, Johnson \& Johnson, Hanmi and MannKind, and Novartis. No other potential conflicts of interest relevant to this article were reported.

Compliance with Ethical Guidelines. This study is a subanalysis of primary EDGE publication. The study protocol for EDGE was reviewed and approved by all local independent ethics committees or institutional review boards (IRBs). All procedures followed were in accordance with the ethical standards of the committee on human experimentation and with the Declaration of Helsinki of 1964, as revised in 2013, and Good Clinical Practice Guidelines. Written informed consent was obtained from all patients prior to inclusion in the study.

Data Availability. The datasets analyzed during the current study are available from the corresponding author upon reasonable request.

Open Access. This article is distributed under the terms of the Creative Commons Attribution-NonCommercial 4.0 International License (http://creativecommons.org/licenses/ by-nc/4.0/), which permits any noncommercial use, distribution, and reproduction in any medium, provided you give appropriate credit to the original author(s) and the source, provide a link to the Creative Commons license, and indicate if changes were made.

\section{REFERENCES}

1. American Diabetes Association. Approaches to glycemic treatment. Diabetes Care. 2016;39(Suppl. 1):S52-9.

2. Singh AK. Deciding oral drugs after metformin in type 2 diabetes: an evidence-based approach. Indian J Endocrinol Metab. 2014;18:617-23.

3. Ahrén B, Foley JE. Improved glucose regulation in type 2 diabetic patients with DPP- 4 inhibitors: focus on alpha and beta cell function and lipid metabolism. Diabetologia. 2016;59:907-17.

4. Ferrannini E, Fonseca V, Zinman B, et al. Fifty-two-week efficacy and safety of vildagliptin vs. glimepiride in patients with type 2 diabetes mellitus inadequately controlled on metformin monotherapy. Diabetes Obes Metab. 2009;11:157-66.

5. Foley JE, Sreenan S. Efficacy and safety comparison between the DPP-4 inhibitor vildagliptin and the sulfonylurea gliclazide after two years of monotherapy in drug-naïve patients with type 2 diabetes. Horm Metab Res. 2009;41:905-9.

6. Filozof C, Gautier JF. A comparison of efficacy and safety of vildagliptin and gliclazide in combination with metformin in patients with type 2 diabetes inadequately controlled with metformin alone: a 
52-week, randomized study. Diabet Med. 2010;27:318-26.

7. Matthews DR, Dejager S, Ahren B, et al. Vildagliptin add-on to metformin produces similar efficacy and reduced hypoglycaemic risk compared with glimepiride, with no weight gain: results from a 2-year study. Diabetes Obes Metab. 2010;12:780-9.

8. Ahrén B, Mathieu C, Bader G, Schweizer A, Foley JE. Efficacy of vildagliptin versus sulfonylureas as add-on therapy to metformin: comparison of results from randomised controlled and observational studies. Diabetologia. 2014;57:1304-7.

9. Sahay BK, Seshiah V. Importance of observational studies in understanding regional clinical practice: rationale and design of the A1chieve study. J Assoc Physicians India. 2013;61:6-8.

10. Treweek S, Zwarenstein M. Making trials matter: pragmatic and explanatory trials and the problem of applicability. Trials. 2009;10:1-9.

11. Kahn SE, Haffner SM, Heise MA, et al. Glycemic durability of rosiglitazone, metformin, or glyburide monotherapy. N Engl J Med. 2006;355:2427-43.

12. UK Prospective Diabetes Study (UKPDS) Group. Intensive blood-glucose control with sulphonylureas or insulin compared with conventional treatment and risk of complications in patients with type 2 diabetes (UKPDS 33). Lancet. 1998;352:837-53.

13. Mathieu $\mathrm{C}$, Barnett $\mathrm{AH}$, Brath $\mathrm{H}$, et al. Effectiveness and tolerability of second-line therapy with vildagliptin vs. other oral agents in type 2 diabetes: a real-life worldwide observational study (EDGE). Int J Clin Pract. 2013;67:947-56.

14. Holman RR. Assessing the potential for alpha-glucosidase inhibitors in prediabetic states. Diabetes Res Clin Pract. 1998;40:S21-5.

15. Lajara R, Aguilar R, Hehnke U, Woerle HJ, von Eynatten M. Efficacy and safety of linagliptin in subjects with long-standing type 2 diabetes mellitus ( $>10$ years): evidence from pooled data of randomized, double-blind, placebo-controlled, phase III trials. Clin Ther. 2014;36:1595-605.

16. Kozlovski P, Bhosekar V, Foley JE. DPP-4 inhibitor treatment: $\beta$-cell response but not HbA1c reduction is dependent on the duration of diabetes. Vasc Health Risk Manag. 2017;13:123-6.

17. Yusta B, Baggio LL, Estall JL, et al. GLP-1 receptor activation improves beta cell function and survival following induction of endoplasmic reticulum stress. Cell Metab. 2006;4:391-406.

18. Mamza J, Mehta R, Donnelly R, Idris I. Important differences in the durability of glycaemic response among second-line treatment options when added to metformin in type 2 diabetes: a retrospective cohort study. Ann Med. 2016;48:224-34.

19. Moen MF, Zhan M, Hsu VD, et al. Frequency of hypoglycemia and its significance in chronic kidney disease. Clin J Am Soc Nephrol. 2009;4:1121-7.

20. Tsuprykov O, Ando R, Reichetzeder C, et al. The dipeptidyl peptidase inhibitor linagliptin and the angiotensin II receptor blocker telmisartan show renal benefit by different pathways in rats with $5 / 6$ nephrectomy. Kidney Int. 2016;89:1049-61.

21. Sharkovska Y, Reichetzeder C, Alter M, et al. Blood pressure and glucose independent renoprotective effects of dipeptidyl peptidase- 4 inhibition in a mouse model of type- 2 diabetic nephropathy. J Hypertens. 2014;32:2211-23.

22. Alter ML, Ott IM, von Websky K, et al. DPP-4 inhibition on top of angiotensin receptor blockade offers a new therapeutic approach for diabetic nephropathy. Kidney Blood Press Res. 2012;36:119-30.

23. Gregg EW, Li Y, Wang J, et al. Changes in diabetes-related complications in the United States, 1990-2010. N Engl J Med. 2014;370:1514-23.

24. International Diabetes Federation. IDF Diabetes Atlas. 7th edn. Brussels, Belgium; 2015. https:// www.idf.org/e-library/welcome.html. Accessed 10 May 2017. 